International Journal of Computer Networks \& Communications (IJCNC) Vol.7, No.2, March 2015

\title{
Energy Efficient Clustering in Heterogeneous Wireless Sensor Networks Using Degree of CONNECTIVITY
}

\author{
Ajay Sikandar ${ }^{1}$ and Sushil Kumar ${ }^{2}$ \\ School of Computer and Systems Sciences,Jawaharlal Nehru University,New Delhi- \\ 110067
}

\begin{abstract}
Cluster head election is a key technique used to reduce energy consumption and enhancing the throughput of wireless sensor networks. In this paper, a new energy efficient clustering (E2C) protocol for heterogeneous wireless sensor networks is proposed. Cluster head is elected based on the predicted residual energy of sensors, optimal probability of a sensor to become a cluster head, and its degree of connectivity as the parameters. The probability threshold to compete for the role of cluster head is derived. The probability threshold has been extended for multi-levels energy heterogeneity in the network. The proposed E2C protocol is simulated in MATLAB. Results obtained in the simulationshowthat performance of the proposed E2Cprotocol is betterthan stable election protocol (SEP), and distributed energy efficient clustering (DEEC) protocol in terms of energy consumption, throughput, and network lifetime.
\end{abstract}

\section{KEYWORDS}

Cluster Head Election, Degree of Connectivity, Residual Energy, Energy Heterogeneity, Wireless sensor Network

\section{INTRODUCTION}

Wireless sensor networks (WSNs) consist of large number of tiny and limited battery powered sensors. Sensors monitor the physical environment in a specified region; the sensed data are fused, and forwardedusing multi-hops to the sink. WSNs have wide range of applications such as security surveillance, traffic surveillance, environment monitoring, and healthcare. Such networks have ability to operate without human interaction in a harsh environment. Once the sensors are deployed in such environment, their batteries cannot be recharged or replaced. Therefore, to prolong the network lifetime, energy efficient algorithm need to be developed [1,2]. Due to their limited battery power, energy consumption is a key issue in designingthe network protocols and applications of WSNs. Energy is consumed in WSNs at three levels: sensing, transmission and network operation/processing. The major part of energy is consumed in transmission whereas sensing and operational cost of the network is minimal [3-5].

Grouping sensors into clusters has been pursued in order to gather sensed data,and then fuse at the cluster head. Cluster head election is the key for minimization of energy consumption. Researchers face difficulties in designing cluster protocols for WSNs due to dynamic and complex nature of network topology. A clustering protocol is designed in such a way that energy can be saved and reliable data transmission is achieved. All members transmit data to their cluster heads, which forward data to sink after data aggregation. It reduces redundancy in the data which, in turn, minimize the number of transmissions thus saving energy and bandwidth resources [6,7].

DOI:10.5121/ijcnc.2015.7202 
International Journal of Computer Networks \& Communications (IJCNC) Vol.7, No.2, March 2015

Clustered WSNs can be classified into two categories: homogeneous and heterogeneous networks. A homogeneous WSN is composed of tiny, resource constrainedsensors having the same hardware capabilities. A heterogeneous WSN consists of sensors with different energy levels and functionalities. In the network, those sensors are farthest from the cluster head sensor always spend more energy as compare to the sensors that are closer to the cluster heads. The cluster head election is one of the key problems in sensor network applications and can consume significantly more energy for networks communication.This problem can be resolved considering the degree of connectivityand residual energy of a sensor while electing it as cluster head.The sensor is elected as a cluster head if it has highest node connectivity and residual energy. We are motivated by the fact that extra battery energy can be embedded in some of the cluster head sensors. The lifetime of the sensor network is a main problem that requires special attention, hence, to extend the network lifetime, addition of sensors having more energy can be a better option[ $[8,9,10,23,24]$.

In this paper, a novel clustering protocol, Energy Efficient Clustering (E2C), has been for multilevels energy heterogeneous wireless sensor networks. A new cluster head election strategy has been presented. The formula of the cluster head election probability threshold has been derived. E2C uses the residual energy, optimal probability of a sensor to become a cluster head, and degree of sensors as the parameters to elect a sensor as cluster head. The simulation results have been compared with the existing clustering protocols: SEP and DEEC.

The rest of the paper is organized as follows: In section 2 we present the overview of the related work. In section 3, the detail of the proposed protocol is presented. Section 4 presents the simulation results and discussion. Finally we conclude paper in section 5.

\section{RELATED WORK}

Clustering in WSN is a method to organize and manage the network effectively. In [11], authors suggested an energy efficient distributed clustering algorithm which defines suitable cluster sizes depending on the hop distance to the sink. Energy efficient clustering algorithm effectively controls the cluster size which allows an appropriately uniform use of the overall energy. In [12], authors propose a stable election protocol (SEP) for a heterogeneous WSN to prolong the time interval before the death of the first sensor. SEP and energy efficient heterogeneous clustering scheme are based on weighted election probabilities of each sensor to become cluster head according to the remaining energy in each sensor.

Authors suggested an energy efficient prediction clustering algorithm for better performance of network in [13]. It is a new model with heterogeneity of monitored objects and energy heterogeneity of all sensors. To achieve the balance of energy consumption among non-uniform sensors and to prolong network lifetime, authors suggested a clustering based routing protocol for wireless sensor networks with non-uniform node distribution whose cores are an energy aware clustering algorithm in [14]. In [15] authors advise heterogeneous network model with energy heterogeneity and computational heterogeneity according to clustering structure are given in [16].

In [17] authors suggested an energy efficient multilevel heterogeneous routing protocol for WSNs. Operation of multilevel heterogeneity is analyzed by k-level of sensor heterogeneity using suitable heterogeneity parameters. A variable threshold is defined as the ratio of number of alive sensors for current round to the total number of sensors. The threshold is used to elect the cluster headsensors. Authors in [18] implemented a distributed energy-efficient clustering algorithm to 
International Journal of Computer Networks \& Communications (IJCNC) Vol.7, No.2, March 2015

improve coverage of wireless sensor networks. They suggested a clustering technique with the least number of cluster heads to cover the whole network. It periodically updates cluster heads according to the joint information of sensor's residual energy. From the perspective of energy consumption minimization, authors presented energy efficient solution for data forwarding using cooperative communication because of it is more desirable in harsh environment with long haul distance [19].

In [20] authors proposed hybrid energy efficient distributed clustering algorithm. It elects cluster head according to sensor's residual energy and a secondary parameter such as sensor proximity to its neighbor or node degree. In [21] authors designed and evaluated a distributed energy efficient clustering scheme for heterogeneous wireless sensor network. In this scheme, cluster head is elected on the basis of probability of the ratio between residual energy of each sensor and average energy of the network.

\section{E2CPRotocoL}

In this section, we describe the details of architecture of E2C and discuss how efficiently it improves the network lifetime considering the different metrics such as node degree and residual energy of a sensor that is being elected as cluster head.

\subsection{Network and Energy Model Assumption}

We consider the following assumptionsto describe the network model used in this work.

1. Sensors and sinks are stationary after deployment.

2. There are $n$ sensors uniformly dispersed with in a square field of area $M \times M$ square meters.

3. Sink is located at the center of the network field.

4. Sensorsare continuously forwarding data to its appropriate cluster head and cluster head sends data to the sink.

5. Sensors have heterogeneity in term of energy and node degree. Sensors have different initial energy.

6. Sink is not energy limited in comparison to energy of the other sensor in the network.

We calculated the energy consumed for transmitting or receiving messages by adopting the radio dissipation model given in [16]. In this model, energy consumed in the transmission of sensor depends on the sum of constant electronic components energy consumption and amplifier energy proportional to distance between sender and receiver sensors. In the transmission of al bits message energy consumed by radio is given by

$$
E_{T x}(l, d)= \begin{cases}l\left(\varphi+\alpha d^{2}\right) & d \leq d_{0} \\ l\left(\varphi+\beta d^{4}\right) & d>d_{0}\end{cases}
$$

where $\varphi$ is constant energy by electronic components, $\alpha$ and $\beta$ are the amount of energy per bit dissipated in the transmitter amplifier. $d$ is the distance between sender and receiver.The energy consumed $\mathrm{E}_{\mathrm{Rx}}(\mathrm{l})$ to receive thel bit message is given by

$$
\mathrm{E}_{\mathrm{Rx}}(\mathrm{l})=\mathrm{l} \times \varphi
$$


International Journal of Computer Networks \& Communications (IJCNC) Vol.7, No.2, March 2015

\subsection{Optimal Clustering}

We assume that distance of any sensor to the sink or it's cluster head is less than or equal to thresholdvalued $_{0}$. Total energy dissipated in the network per round is equal to

$$
\mathrm{E}_{\text {total }}=\mathrm{l}\left(2 \mathrm{n} \varphi+n \mathrm{E}_{\mathrm{da}}+\alpha\left(\mathrm{kd}_{\mathrm{s}}^{2}+\mathrm{nd}_{\mathrm{CH}}^{2}\right)\right)
$$

where $E_{\mathrm{da}}$ is representing energy consumed in data aggregation, and $\mathrm{k}$ is the number of cluster

heads. $d_{s}$ is the average distance between the cluster head and the sink, and $d_{C H}$ isthe average distance between member sensors in cluster and cluster head. The distance $\mathrm{d}_{\mathrm{CH}}$ can be expresses

$$
\mathrm{d}_{\mathrm{CH}}^{2}=\int_{0}^{\mathrm{xmax}} \int_{0}^{\mathrm{ymax}} \rho(\mathrm{x}, \mathrm{y}) \mathrm{dxdy}
$$

where $\rho(x, y)$ is the node distribution. The average distance between cluster head to sink is given by

$$
d_{\text {to BS }}=\int_{\mathrm{A}} \frac{\sqrt{\mathrm{x}^{2}+\mathrm{y}^{2}}}{4 \mathrm{a}^{2}} \mathrm{dA}=0.765 \mathrm{a}
$$

Where $\mathrm{a}=\mathrm{M} / 2$. By differentiating $\mathrm{E}_{\text {total }}$ with respect to $\mathrm{k}$ and equating it to zero,optimal number of cluster heads can be calculated as

$$
\mathrm{k}_{\mathrm{opt}}=\frac{1}{0.765} \sqrt{\frac{2 \mathrm{n}}{\pi}} \sqrt{\frac{\alpha}{\beta}}
$$

Any sensor can be chosen as cluster head. The optimal probability of a sensor to become a cluster head is expresses as

$$
\mathrm{P}_{\mathrm{opt}}=\frac{1}{0.765} \sqrt{\frac{2}{\mathrm{n} \pi}} \sqrt{\frac{\alpha}{\beta}}
$$

The optimal probability for a sensor to become cluster head is a key aspect. Authors in [9] mentioned that if clusters are not arranged in an optimal way, total energy consumed per round increases exponentially.

\subsection{Cluster Head Election}

In LEACH protocol, all sensors have the same initial energy. It guarantees that every one of them becomes cluster head exactly once every $1 / \mathrm{P}_{\text {opt }}$ round. Let $\mathrm{P}_{\mathrm{i}=} 1 / \mathrm{r}_{\mathrm{i}}$ which can be considered as the average probability of sensors to become cluster head during $\mathrm{r}_{\mathrm{i}}$ round. There are average $\mathrm{n} * \mathrm{P}_{\mathrm{opt}}$ cluster head per round and all sensors die approximately at the same time. Suppose that $r_{i}$ be the number of rounds to become cluster head for $\operatorname{sensorb}_{i}(i=1,2, \ldots n)$. For the homogeneous sensors, choosing the average probability $\mathrm{P}_{\mathrm{i}}$ equal to $\mathrm{P}_{\text {opt }}$ can ensure that there $\operatorname{are}_{\mathrm{opt}} * \mathrm{n}$ cluster headsin every round, and energy of all sensors will be depleted at same time. InHWSNs, we consider that a cluster head sensorhas more energy and higher node degree than non-cluster head sensors. Since the energy of a cluster head is depleted quickly as compare to non-cluster head sensors, this requires that each sensoracts its turn as a cluster head. The average energy at round $\mathrm{k}$ of the network can be calculated as 


$$
\overline{\mathrm{E}}(\mathrm{k})=\frac{1}{\mathrm{~N}} \sum_{\mathrm{i}=1}^{\mathrm{N}} \mathrm{E}(\mathrm{k})
$$

Let us consider that all sensors have the same transmission range $r_{0}$ randomly uniformly placed in a large area A. Sensors are deployed with density $\rho=n / A$ in the network area. Assume that a sensoris placed at a point $(0,0)$ and another sensor is randomly placed according to some arbitrary probability density functionf $\mathrm{X}_{\mathrm{X}}\left(\mathrm{x}^{\prime}\right)$. Thesetwo sensors established a link if second sensoris placed within the transmission range of first sensor. Assume that coverage area of a sensor is denoted by $\mathrm{A}_{0}$. The probability of the link betweentwo sensors is expressed as [22]

$$
\begin{aligned}
P_{0} & =\iint_{A_{0}} f_{X}\left(x^{\prime}\right) d x^{\prime} \\
& =\int_{-r_{0}}^{r_{0}} \int_{-\sqrt{r_{0} y^{\prime 2}}}^{\sqrt{r_{0} y^{\prime 2}}} f_{X}\left(x^{\prime}, y^{\prime}\right) d x^{\prime} d y^{\prime}
\end{aligned}
$$

The degree of a sensoris defined as the number of links it has. The probability that a sensor has degree $d_{k}$ is given by

$$
P\left(d_{k}\right)=\left(\begin{array}{c}
n-1 \\
d_{k}
\end{array}\right) P_{0}^{d_{k}}\left(1-P_{0}\right)^{n-d_{k}-1}
$$

where $P\left(d_{k}\right)$ denotes the probability that asensor has degree $d_{k}$ in round $k$. In the network, each sensor should have the knowledge of energy of all its neighboring sensors. A sensor with more residual energy and maximum degree has higher probability of becoming cluster head after the operation of current round. Using equation (8) and equation (10), the probability for sensorb $b_{i}$ to become cluster head is

$$
\left.\mathrm{P}_{\mathrm{i}}=\mathrm{P}_{\mathrm{opt}} * \frac{\mathrm{E}_{\mathrm{i}}(\mathrm{k})}{\overline{\mathrm{E}}(\mathrm{k})} * \mathrm{P}\left(\mathrm{d}_{\mathrm{k}}\right)\right)
$$

Each sensorb $b_{i}$ becomesa cluster head for the current round if arandom number is drawn between 0 and 1 , and is less than the following probability threshold.

$$
\mathrm{T}\left(\mathrm{b}_{\mathrm{i}}\right)= \begin{cases}\frac{\mathrm{P}_{\mathrm{i}}}{1-\mathrm{P}_{\mathrm{i}}\left(\mathrm{k} \bmod \frac{1}{\mathrm{P}_{\mathrm{i}}}\right)} & \text { if } \mathrm{b}_{\mathrm{i}} \varepsilon \mathrm{G} \\ 0 & \text { otherwise }\end{cases}
$$

where $\mathrm{G}$ is the set of sensors that are eligible to be cluster head at round $\mathrm{k}$.

\subsection{Cluster Head Election with Heterogeneous Sensors}

In this section, we calculate the probability of electing a sensor as a cluster head in the case of multilevel heterogeneity. Different initial energy levels for different types of sensors are considered as heterogeneity factor. First we derive the probability ofelecting a sensor as a cluster head with two types of sensors: normal and advancesensors.Assume that there are $\mathrm{m}$ advance sensors and $n(1-m)$ normal sensors. The energy of each advance sensorsis $(1+a)$ times more than the energy of each normal sensor, where a is the energy factor. The total energy of the network is increased by a factor of $(1+a m)$. The weight is defined as the ratio of the initial energy of a sensor to the initial energy of normal sensor. The average number of cluster heads 
from normal sensors equal to $\mathrm{P}_{\mathrm{opt}} \mathrm{n}=\mathrm{P}_{\mathrm{nrm}} \mathrm{n}(1+\mathrm{am})$, where $\mathrm{P}_{\mathrm{nrm}}$ is the weighted election probability for normal sensor. The weighted election probabilities of normal and advanced sensor are given by[12]

$$
\left\{\begin{array}{l}
\mathrm{P}_{\mathrm{nrm}}=\frac{\mathrm{P}_{\mathrm{opt}}}{(1+\mathrm{am})} \\
\mathrm{P}_{\mathrm{adv}}=\frac{(1+\mathrm{a}) \mathrm{P}_{\mathrm{opt}}}{(1+\mathrm{am})}
\end{array}\right.
$$

Now, we define the probability $P_{i}$ to become a cluster head from normal and advanced sensors in two case of heterogeneity as

$P_{i}=\left\{\begin{array}{lc}\frac{P_{o p t}}{(1+a m)} * \frac{E_{i}(k}{\bar{E}(k)} * P\left(d_{i}\right) & \text { For normal sensors } \\ \frac{(1+a) P_{o p t}}{(1+a m)} \frac{E_{i}(k}{\bar{E}(k)} * P\left(d_{i}\right) & \text { For advanced sensors }\end{array}\right.$

Now, we introduce third type of sensors: super sensors in the network.Let $\mathrm{m}_{0}$ is the percentage of the total number of sensorsmhaving $b$ times more energy than the energy of the normal sensors. The total energy of the network is increased by a factor of $\left(1+m\left(a+m_{0} b\right)\right)$. The weighted election probabilities for these three types of sensors: normal, advanced and super sensoras [9]

$$
\left\{\begin{array}{l}
\mathrm{P}_{\mathrm{nrm}}=\frac{\mathrm{P}_{\mathrm{opt}}}{\left(1+\mathrm{m}\left(\mathrm{a}+\mathrm{m}_{0} \mathrm{~b}\right)\right)} \\
\mathrm{P}_{\mathrm{adv}}=\frac{(1+\mathrm{a}) \mathrm{P}_{\mathrm{opt}}}{\left(1+\mathrm{m}\left(\mathrm{a}+\mathrm{m}_{0} \mathrm{~b}\right)\right)} \\
\mathrm{P}_{\text {sup }}=\frac{(1+\mathrm{b}) \mathrm{P}_{\mathrm{opt}}}{\left(1+\mathrm{m}\left(\mathrm{a}+\mathrm{m}_{0} \mathrm{~b}\right)\right)}
\end{array}\right.
$$

Similarly, we define the probability $P_{i}$ to become a cluster head from normal, advanced and super sensors in three case of heterogeneity as

$$
\mathrm{P}_{\mathrm{i}}=\left\{\begin{array}{lr}
\frac{\mathrm{P}_{\mathrm{opt}}}{\left(1+\mathrm{m}\left(\mathrm{a}+\mathrm{m}_{0} \mathrm{~b}\right)\right)} * \frac{\mathrm{E}_{\mathrm{i}}(\mathrm{k}}{\overline{\mathrm{E}}(\mathrm{k})} * \mathrm{P}\left(\mathrm{d}_{\mathrm{i}}\right) & \text { for normal sensors } \\
\frac{(1+\mathrm{a}) \mathrm{P}_{\mathrm{opt}}}{\left(1+\mathrm{m}\left(\mathrm{a}+\mathrm{m}_{0} \mathrm{~b}\right)\right)} * \frac{\mathrm{E}_{\mathrm{i}}(\mathrm{k}}{\overline{\mathrm{E}}(\mathrm{k})} * \mathrm{P}\left(\mathrm{d}_{\mathrm{i}}\right) & \text { for advanced sensors } \\
\frac{(1+\mathrm{b}) \mathrm{P}_{\mathrm{opt}}}{\left(1+\mathrm{m}\left(\mathrm{a}+\mathrm{m}_{0} \mathrm{~b}\right)\right)} * \frac{\mathrm{E}_{\mathrm{i}}(\mathrm{k}}{\overline{\mathrm{E}}(\mathrm{k})} * \mathrm{P}\left(\mathrm{d}_{\mathrm{i}}\right) & \text { for super sensors }
\end{array}\right.
$$

We can generalizeit for multilevel heterogeneous network. The probability of a sensor to be a cluster head in case of multilevel heterogeneity is given by

$P_{i}=\frac{N * P_{o p t}\left(1+a_{i}\right)}{\left(N+\sum_{i=1}^{N} a_{i}\right)} * \frac{E_{i}(k)}{\bar{E}(k)} * P\left(d_{i}\right)$

Each sensor to become a cluster head for current round can be determined from equation (12) for equations (14), (16), and (17). Thus, the probability threshold value of cluster head depends on the residual energy, initial energy, and degree of a sensor. The cluster heads act as local control center to manage the data transmission in their cluster. Cluster heads transmit TDMA schedule to the sensors in their cluster. This makes sure that there is no collision among the transmission of data messages. Cluster heads allow radio component of each non cluster head sensor to be turnoff 
at all-time except during transmission time, thus minimizing energy consumption of individual sensor of the network.

\section{Simulation RESUltSAND DiscuSSION}

In this section, we simulatedtheE2C protocol in MATLAB and the results obtained in the simulation have been compared with the exiting clustering protocols: SEP, and DEEC.In the simulation, the network area of $100 \times 100 \mathrm{~m} 2$ has been taken for heterogeneous clustered wireless sensor network. Different types of sensors having different level of energy are distributed over the network area. It is assumed that sink node is placed at the center of network area. The distance between sensors and sink is assumed to be $70 \mathrm{~m}$. We neglect the effect caused by signal collision and interference in wireless channel. In HWSNs, we used radio parameters mentioned in Table 1. The simulations have been carried out for the cases of two, three and multi-types of sensors having two, three and multi-levels of energy correspondingly in heterogeneous WSNs.

Table1. Simulation Parameter

\begin{tabular}{|l|l|}
\hline $\mathrm{n}$ & 100 \\
\hline $\mathrm{M} \times \mathrm{M}$ & $100 \times 100 \mathrm{~m} 2$ \\
\hline $\mathrm{E}_{\mathrm{da}}$ & $5 \mathrm{nj} / \mathrm{bit}$ \\
\hline$\varphi$ & $50 \mathrm{nj} / \mathrm{bit}$ \\
\hline$\alpha$ & $10 / \mathrm{nj} / \mathrm{bit} / \mathrm{m} 2$ \\
\hline$\beta$ & $0.0013 \mathrm{pj} / \mathrm{bit} / \mathrm{m} 4$ \\
\hline Message size & $5000 \mathrm{bits}$ \\
\hline Initial energy of normal sensor & $0.5 \mathrm{j}$ \\
\hline$P_{\text {opt }}$ & 0.1 \\
\hline
\end{tabular}

\subsection{Heterogeneity with two levels of energy}

In this case, 25 advance sensorshave been deployed with 2 times energy that of the normal sensors. The numbers of normal sensors deployed are 75 . The value of the rest simulation parameters are given in table -1 . Figure 1 depicts total number of sensors that remain alive over the simulation time period.The first sensor dies at after rounds 2050 and the last sensor dies around at 5700 rounds. For the round 2700 the number of alivesensorsof E2Cis same as that of SEP. For rounds between 2050and 2700, it is observedthatthe number of alivesensor is more for E2C as compared toSEP and DEEC. After round 2700, the SEP performs better than E2C. Additionally, E2C performsbetter than DEEC. It is observed that overall performance of E2C is better as compared to SEP and DEEC.It is because it elects a cluster head usingthe residual energy, and degree of the sensors, and makesthe optimum number of cluster heads.

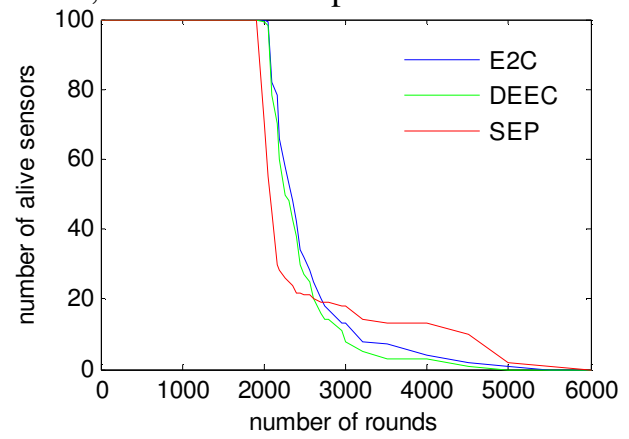

Figure1. Number of alive sensors over rounds under heterogeneity with two level of energy of SEP, DEEC and $\mathrm{E} 2 \mathrm{C}$ 
Figure 2 illustrates that number of received data packets at sink verses the simulation time period. The result obtained in the simulation shows that the number of packets received by sink of the proposed protocol E2C is increasing rapidly as compared to DEEC and SEP when the number of rounds is less 2500. It is because the cluster head sensors of the E2C are better connected than that of DEEC and SEP. For rounds between 2500 and 6000, growth in the received packets at sink is constant. This can be attributed the fact that the number of cluster heads per round is stable. When there are no cluster head selected in some rounds, the data packet cannot be transmitted to sink. As a result, the data packets received at the sink per round of E2C is more than that of DEEC and SEP.

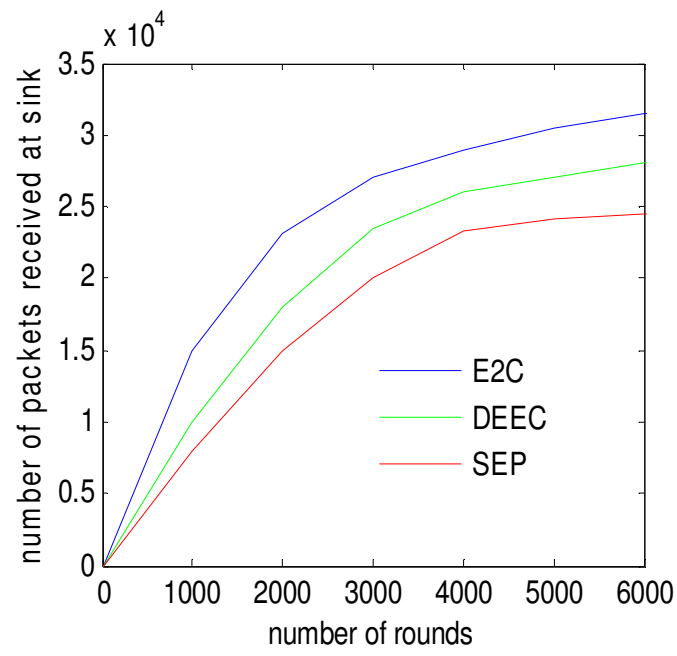

Figure 2. Data packets received over rounds under heterogeneity with two level of energy of SEP, DEEC and $\mathrm{E} 2 \mathrm{C}$

Figure 3 shows the total residual energy of network over simulation time period for different clustering protocols. In the simulation, total initial energy of the network was 85 joules. The simulation is performed for 6000 rounds. The total energy of network is almost depleted upto 4000 rounds for all the protocols considered in the simuation. From the result, it is observed that the total residual energy of E2C protocol is more than that of SEP and DEEC protocols in the same number of rounds. This due the fact that the sensors with more residual energy and higher degree have higher probability of becoming cluster heads.

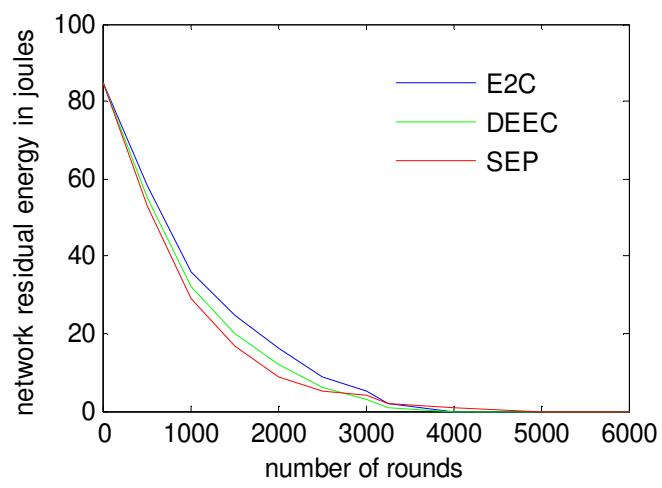

Figure3. The total residual energy of network over rounds under heterogeneity with two level of energy of SEP, DEEC and E2C 


\subsection{Heterogeneity with three levels of energy}

In this case, 20 advance sensors and 30 super sensors have been deployed consisting of 1.5 and 2.5 times more energy than normal sensors correspondingly. The numbers of normal sensors deployed are 50. The value of the rest simulation parameters are given in table -1 .

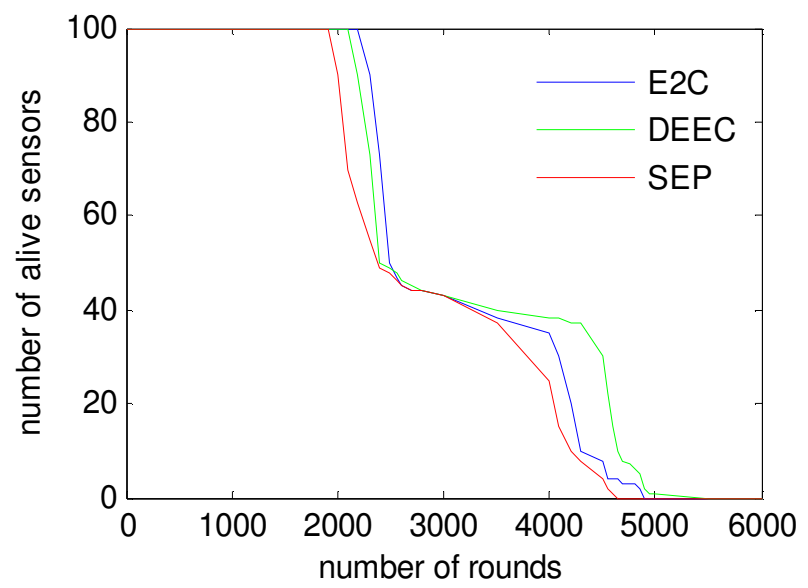

Figure4. Number of alive sensors over rounds under heterogeneity with three levels of energy of SEP, DEEC and E2C

Figure 4 shows the total number of sensors alive over simulation time period. In the $\mathrm{E} 2 \mathrm{C}$, the first sensor dies at rounds 2200 and last sensor dies around at round 5100. From rounds 2400 to 4500, it is observed that the number of dead sensorsis very small.The sensor death rate of SEP and DEEC is substantial as compared to E2C. Thesensors remain alive for longer duration in E2Cas compared with SEP and DEEC.

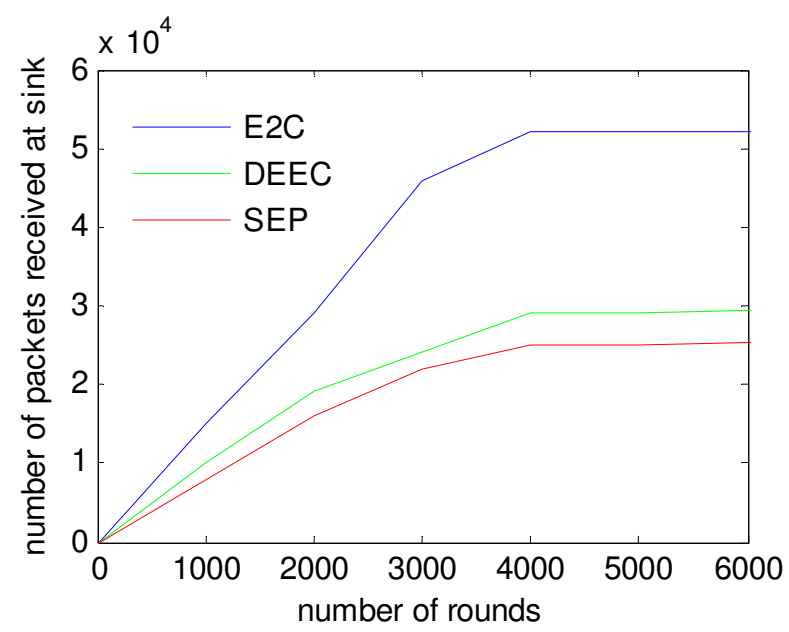

Figure 5. Data packets over rounds under heterogeneity with three levels of energy of SEP, DEEC and E2C

Figure 5 represents the number of data packets received at sink verses simulation time period. The result obtained in the simulation shows that the number of packets received by sink of E2C is increasing rapidly as compared to DEEC and SEP when the number of rounds is less 4000. It is because the cluster head sensors of E2C are elected considering the degree of sensors as an 
additional parameter. Between the rounds 4000 to 6000 , the number of data packets received at sink is almost constant. This is due to the fact that sensor field becomes sparse andthe growth in cluster head election is became constant. As the result, it is observed that data packets received at sink per round for E2C is more as compared to DEEC and SEP.

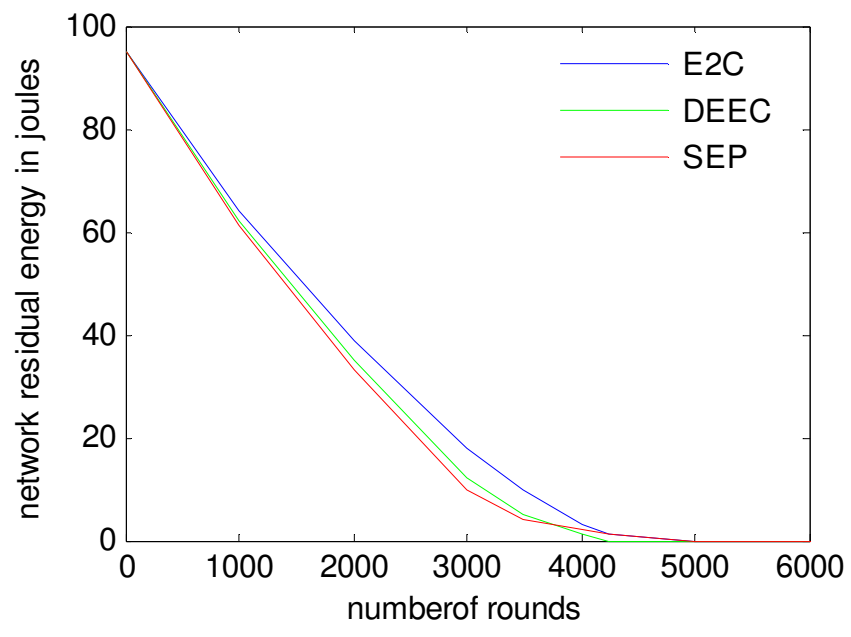

Figure6.Totalresidual energy of network over rounds under heterogeneity with three levels of energy of SEP, DEEC and E2C

Figure6 showstotal residual energy of networkover simulation time period.In the beginning of the simulation, the total residual energy of network was 95 joules. From the result, it is clear thatthe total residual energy of E2C is better than that of SEP and DEEC. This due the fact that death of sensors changes the degree of connectivity, which increases the probability of becoming cluster head having higher degree after the current round.

\subsection{Heterogeneity with multi-levelsenergy}

In this case, the initial energy of sensors is randomly distributed with in the set $[0.5,4.0]$.The value of the rest simulation parameters are given in table -1 .

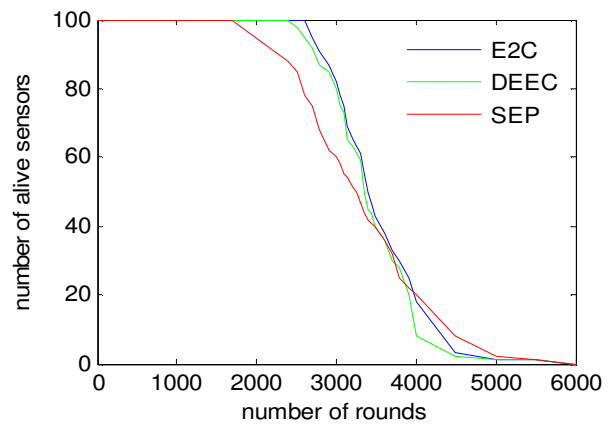

Figure7. Number of alive nodes over rounds under heterogeneity with multi-levelsenergy of SEP, DEEC and $\mathrm{E} 2 \mathrm{C}$

Figure 7 depicts that total number of sensors remains alive verses simulation time. In the E2C, that first sensor dies in 2550 round and last sensor dies in 5500 round. All the protocols have the same number of alive sensors at rounds 3500. As result, it is observed that the number of alive 
sensors is more than that of SEP and DEEC in all rounds. Figure 8 depicts that the number of data packets received by sink over simulation time period. In E2C, the number of data packet received at sink increases linearly as thenumber of rounds increases upto 3000 rounds in all the protocol considered in the simulation. After 3000 rounds, the increment in the number of received packets declines for SEP and DEEC, however the increment in the received packets for E2C continues. This indicates that thecluster heads election of E2C is better than SEP and DEEC.

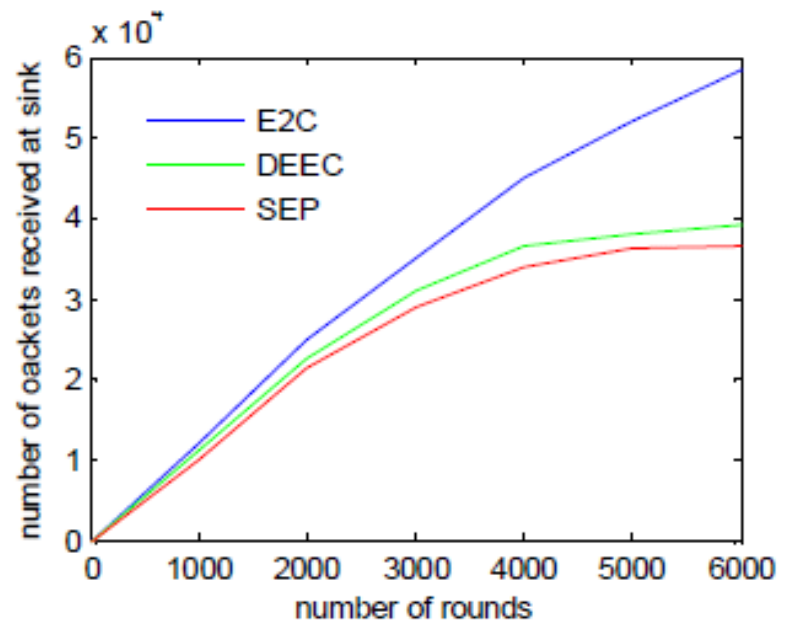

Figure 8. Data Packets over rounds underheterogeneity with multi-levels energy of SEP, DEEC and E2C

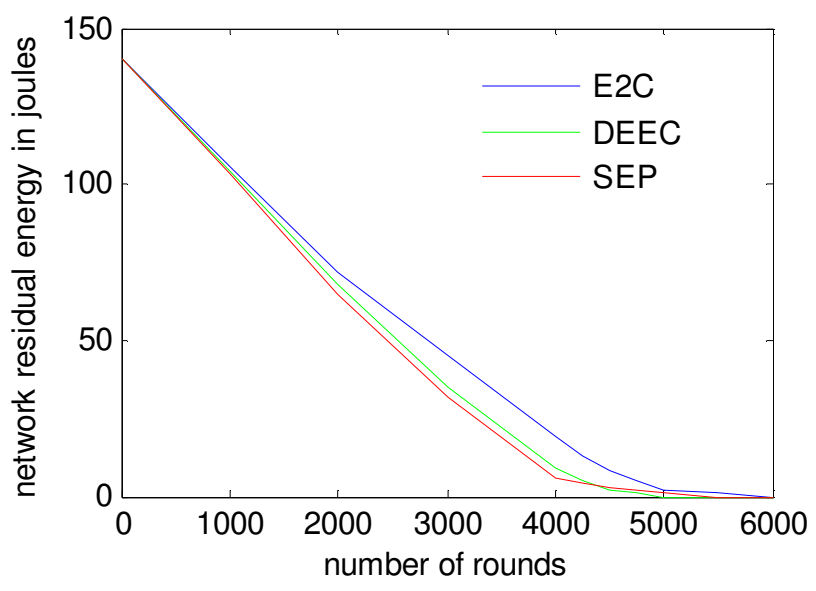

Figure9.Totalresidual energy over rounds under heterogeneity with multi-levels energy of SEP, DEEC and E2C

Figure 9 shows that total initial energy of network was 140 joules. The total energy decrease linearly upto 4900 rounds for all protocols considered in the simulation. The rate of energy dissipation for all sensors in E2C is comparatively less than that of DEEC and SEP. 
International Journal of Computer Networks \& Communications (IJCNC) Vol.7, No.2, March 2015

\section{CONCLuSion}

In this paper, we propose a novel clusteringprotocol,E2C, in which a new cluster head election strategy is developed, which uses the predicted residual energy of sensors, optimal probability of a sensor to become a cluster head, and degree of connectivity as the parameters to contends for the role of the cluster head. The mathematical model for electing the cluster head has been presented. Multi-levels energy heterogeneity in the network has been considered. E2C improvesin energy saving of the heterogeneous wireless sensor network and prolongsthe lifetimeof thenetwork. Proposed protocol was simulated on MATLAB platform. Analysis of simulation results show thatthe performance of E2Cprotocol is better than the considered state of the art protocols in terms of total residual energy, the number of data packets received, and the number of alive sensors.

\section{REFERENCES}

[1] I. Akyildz, W. Su, Y. Sankarasubramaniam, \&E. Cayirci, (2000) “A Survey on sensor network", IEEE Communication Magzine, Vol.40, No. 8, pp.102-114.

[2] A. Sikandar, S. Kumar, G. U. K. Murya (2014) "Optimizing delay for MAC in randomly distributed wireless sensor networks", International Conference on advance Computing, Networking and informatics (ICACNI), Springer, Vol.243,pp. 609-618.

[3] G. Anastasi, M. Conti, M. D. Francesco, A. Passarella, (2009) "Energy conservation in wireless sensor networks: A survey", Ad Hoc Networks, Vol. 7, No. 3, pp. 537-568.

[4] A. Sikandar, S. Kumar,(2014) "Performance analysis of channel access model for MAC in randomly distribute4d wireless sensor networks" International Journal of computer network and communication, Aircc,Vol.6 no. 5, pp.99-110.

[5] A. Sikandar, S. Kumar, (2014) "Performance analysis of interference aware power control scheme for TDMA in wireless sensor networks", International Conference on advance Computing, Networking and informatics (ICACNI), Springer, Vol. 28, pp. 95-101.

[6] W. Heinzelman, A. Chandrakasan\&H. balakrishnan, (2000) "Energy-Efficient communication protocol for wireless sensor network",In the Proceeding of the Hawaii international Conference System Sciences, Hawaii, pp. 1-10.

[7] A. A. Abbasi\& M. F. Younis, (2007) "A survey on clustering algorithms for wireless sensor networks", Computer Communications, Vol. 30, No. (14-15), PP. 2826-2841.

[8] V. Mhatre\&C. Rosenberg, (2004) "Homogeneous vs Heterogeneous Clustered Networks: A comparative study", in Proceedings of IEEE international conference on communication (ICC 2004), pp. 1-6.

[9] D. Kumar, T. C. Aseri, R. B. Patel, (2009) "EEHC: Energy efficient heterogeneous clustering scheme for wireless sensor networks", Computer Communication, Elsevier, Vol. 32, No. 4, pp. 662-667.

[10] P. Saini, A. K. Sharma, (2010) “Energy Efficient Scheme for Clustering Protocol Prolonging the Lifetime of Heterogeneous Wireless Sensor Network” International Journal of Computer Application, Vol. 6, No. 2, pp. 30-36.

[11] D. Wei, Y. Jin, S.Vural, K.Moessner\& R. Tafazolli, (2011) “An Energy-Efficient clustering solution for wireless sensor networks", IEEE transactions on wireless communications, Vol. 10, no. 11, pp. 3973-3983.

[12] G. Smaragdakis, I. Matta \&A. Bestavros, (2004) "SEP: A stable election protocol for clustered heterogeneous wireless sensor networks", in second international Workshop on sensor and Actor Network protocols and Application (SANPA), pp.1-11.

[13] J. Peng, T. Liu, H. Li, B. Guo,(2013) "Energy-Efficient prediction clustering algorithm multilevel heterogeneous wireless sensor network", International Journal of Distributed Sensor Networks, Hindawi, Vol.2013, pp.1-7.

[14] J. Yu, Y. Qi, G. Wang \&X, Gu (2012) “A cluster-based routing protocol for wireless sensor networks with non-uniform node distribution", International Journal of Electronic and Communication, Elsevier,pp.54-61. 
[15] H. Zhou, Y. Wu, Y. Hu \& G. Xie,(2010) "A novel stable selection and reliable transmission protocol for clustered heterogeneous wireless sensor network", Computer Communication, Elsevier, Vol. 33,No. 15,pp. 1843-1849.

[16] W. B. Heinzelman, A. P. Chanrakasan, H. Balakrishana, (2002) “An application specific protocol architecture for wireless micro sensor network" IEEE Transection on Wireless Communications, Vol. 1, No. 4, pp. 660-670.

[17] S. Tanwar, N. Kumar \& J. W. Niu,(2014) "EEMHR: Energy-efficient multilevel heterogeneous routing protocol for wireless sensor networks",International Journal of Communication Systems, Wiley, Vol. 27, No.9, pp.1289-1318.

[18] Z. Liu, Q. Zhenga, L. Xuea, X. Guana,(2012) “A distributed energy-efficient clustering algorithm with improved coverage in wireless sensor networks", Future Generation Computer Systems, Elsevier, Vol. 28, No. 5, pp. 780-790.

[19] D. Zhang \& Z. Chen (2013) "Energy-Efficiency of cooperative communication with guaranteed E2E reliability in WSNs", International Journal of Distributed Sensor Network, Hindawi, Vol. 2013, pp.111.

[20] O. Younis, S. Fahmy "HEED: A Hybrid, Energy-Efficient, Distributed Clustering Approach for Ad Hoc Sensor Networks” IEEE Transactions on Mobile Computing, Vol. 3, No. 4,pp.366-369.

[21] L. Qing, Q. Zhu \&M. Wang, (2006) "Design of a distributed energy- efficient clustering algorithm for heterogeneous wireless sensor network", Computer communication, Elsevier, pp.2230-2237.

[22] C. Bettstetter, (2004) "On the connectivity of Adhoc network", the Computer Journal, Special Issue on Mobile and Pervasive Computing, Oxford University Press, Vol. 47, No. 4, pp. 432-447.

[23] R. Tandon, (2012) "Determination of optimal number of clusters in wireless sensor networks", International Journal of Computer Network and Communication, Aircc, Vol. 4, No. 4, pp. 235-249.

[24] S. Kumar, S. Kumar, B. Bhusan, (2014) "Energy aware clustering protocol (EACP) for Heterogeneous WSNs", International Journal of Computer Network and Communication, Aircc, Vol. 6, No. 4, pp.23-37.

\section{Authors}

Ajay Sikandar is currently a Ph.D. research scholar at School of Computer and Systems Sciences, Jawaharlal Nehru University, New Delhi, India. His research interests include Wireless Sensor Networks and Mobile Ad-hoc Networks. He received his MCA and M. Tech degrees in Computer Science and Technology from School of Computer and Systems Sciences, Jawaharlal Nehru University, New Delhi, India in 2003 and 2010, respectively, and B.Sc. degree in mathematics from PurvanchalUniversity Jaunpur, India in 1998.

Sushil Kumar received his Ph.D., M. Tech and MCA degrees in Computer Science from School of Computer and Systems Sciences, Jawaharlal Nehru University, New Delhi, India in 2014, 1999 and 1997 respectively, and B. Sc. degree in Mathematics from Kanpur University, India in 1993. He is currently working as Assistant Professor at School of Computer and Systems Sciences, Jawaharlal Nehru University, New Delhi, India. His research interest includes vehicular ad hoc networks, mobile ad hoc networks and wireless sensor networks. Dr. Kumar has published papers in International Journals and Conferences including ACM, IEEE, Springer, Inderscience, MDPI, and Hindawi. 